\title{
Alemtuzumab in the treatment of fludarabine refractory B-cell chronic lymphocytic leukemia (CLL)
}

\author{
Marco Montillo \\ Francesca Ricci \\ Sara Miqueleiz \\ Alessandra Tedeschi \\ Enrica Morra \\ Department of Oncology/Hematology, \\ Division of Hematology and Bone \\ Marrow Transplant Unit, Niguarda Ca' \\ Granda Hospital, Milan, Italy
}

\begin{abstract}
The introduction of immunotherapeutic agents has provided renewed hope for Chronic lymphocytic leukemia fludarabine-refractory patients. Several clinical trials have shown that alemtuzumab is a more effective option compared to combination chemotherapy for treatment of patients who have relapsed or who are refractory to fludarabine, including those with poor prognostic factors. Although there are significant potential toxicities associated with alemtuzumab, such as infusional reactions and the risk of cytomegalovirus (CMV) reactivation, most are manageable. Pre-treatment anti-pyretics and anti-histamines are recommended to prevent or mitigate the acute infusional reactions associated with intravenous infusion. Recent use of alemtuzumab via the subcutaneous route has been shown to be well tolerated and has yielded similar response rates to the infusional method of administration. Prophylaxis with thrimethoprim/sulphamethoxazole (TMP/SMZ) as well as valacyclovir or a similar anti-viral can prevent many of the opportunistic infections seen in early trials. Reactivation of CMV infection can be effectively managed with monitoring and early treatment. Chemo-immunotherapy combination with alemtuzumab has been tested and demonstrated unprecedented clinical results in relapsed and refractory patients. The use of this agent earlier in the algorithm of patients with these characteristics should be considered. Future areas of research will include the use of alemtuzumab in combination with other monoclonal antibodies and other targeted therapies.
\end{abstract}

Keywords: chronic lymphocytic leukemia, fludarabine, alemtuzumab

\section{Background}

Chronic lymphocytic leukemia (CLL) is a malignancy that results in accumulation of mature, monoclonal B-cells in blood, lymphnodes, spleen and bone marrow producing lymphocytosis, adenopathy, organomegaly and progressive bone marrow failure (Kipps 2001). The basic physiopathologic defect in CLL is resistance of neoplastic cells to programmed-cell death or apoptosis. Median survival of these patients is about 10 years but it largely varies from one patient to another: from less than 3 years to a normal life expectancy. Therefore, treatment should be individualized on the basis of the risk to each patient (Montserrat 2002; Byrd et al 2004).

Historically, the clinical course for the majority of patients with CLL has been predictable, with a response to initial treatment, eventual recurrence, reduced likelihood of response with subsequent treatments, and shorter duration of response with each remission. Retreatment typically yields progressively less satisfactory response than the prior treatment, eventually leading to refractory disease.

For many years alkylating agents-based regimens utilizing chlorambucil (CHL) or cyclophosphamide (CTX) were the mainstays of treatment for CLL which resulted in a complete response (CR) rate in less than 10\% along with palliation of symptoms, and only a modest, if any, impact on survival (Knaspe et al 1974; Oken and Kaplan 1979). 
Clinical research has been aimed at developing new treatments that prolong survival and potentially cure patients with CLL. Response to treatment has been assessed in clinical trials by the National Cancer Institute-Working Group (NCI-WG) criteria published more than 10 years ago (Cheson et al 1996).

A response-driven approach has been commonly used, based on the observation that patients who achieve CR live longer that those who achieve partial remission (PR) or those who fail treatment.

The introduction of purine analogs provided a new treatment option for CLL. Purine analogs have shown increased numbers of CRs compared with alkylating agents, particularly when administered to previously untreated patients. Until the purine analogs were developed, no treatment was available that was effective in CLL patients who were resistant to alkylating agents. Fludarabine (FAMP) has been the most commonly used in this class of drugs, and it was rapidly shown to be capable of achieving a good response rate in patients who were refractory to CHL. In the context of second line therapy, FAMP has been shown to compare favorably to other available treatments in terms of effectiveness, side effects and cost (Grever et al 1985; Keating et al 1989; Saven and Piro 1993).

Three phase III studies have shown that FAMP is superior to CHL and to a combination of CTX, adriamycin, and prednisone (CAP) regarding the number of complete remissions and the duration of remission in first-and secondline treatment of CLL (Johnson et al 1996; Rai et al 2000; Leporrier et al 2001). However, none of these studies could demonstrate a benefit on median overall survival (OS).

The majority of patients who achieve remission following FAMP treatment are known to suffer a relapse after a median of 20-30 months (Rai et al 2000).

It is important to separate refractory from relapsing disease. Whereas treatment of patients with relapsing disease, particularly when the progression-free-interval has been long (eg, $>12$ months), can be envisaged as in newly diagnosed patients, patients with truly refractory disease have an extremely poor prognosis and require immediate intervention. As mentioned before, resistance to $\mathrm{CHL}$ or other alkylating agents at low/standard doses can sometimes be overcome by purine analogs. For example, in the study of Rai et al comparing FAMP to CHL, $46 \%$ of patients failing CHL did respond to FAMP, whereas only $7 \%$ of the 29 patients who were crossed from FAMP to CHL had a response (Rai et al 2000). For patients who become refractory or who demonstrate a primary resistance to FAMP, the prognosis is generally poor (Keating et al 2002a).
Now resistance to FAMP is emerging as a problem in an increasing number of patients. Other approaches combine FAMP with other chemotherapy agents and explore these novel combinations to capitalize on possible synergistic effects. The most effective salvage regimens are considered to be the combinations of purine analogs and CTX. (Keating et al 2002a)

Three recently published phase III trials comparing FAMP and CTX with FAMP alone showed superiority of the combination in untreated patients with B-CLL (Eichhorst et al 2006; Catowsky et al 2007; Flinn et al 2007).

One small report suggested that cladribine (2-CDA) was effective in FAMP refractory patients but this data was disputed by larger series (Juliusson et al 1992; Delannoy et al 1993; O’Brien et al 1994).

Alternative therapies are needed for such patients, preferably using agents whose mechanism of action does not overlap with those of prior chemotherapies. In fact, patients resistant to alkylating agents have a lower response rate to FAMP than sensitive patients, suggesting a final common pathway of resistance (Keating et al 1993).

\section{Alemtuzumab in relapsed/ refractory CLL}

One such approach by monoclonal antibodies is now offered. Alemtuzumab is a humanized monoclonal antibody against CD52, an antigen expressed at high density on most normal and malignant B and T lymphocytes, but not on hematopoietic stem cells (Gilleece et al 1993). Binding of alemtuzumab to CD52 on target cells may cause cell death by 3 different mechanisms: complement activation (Heit et al 1986), antibody-dependent cellular cytotoxicity (Greenwood et al 1993), and apoptosis (Rowan et al 1998).

The difference in clinical results between rituximab, a chimeric human-mouse anti-CD20, and alemtuzumab may be related to the relative expression of CD20 and CD52 on malignant lymphocytes; the level of CD20 expression in patients with B-CLL is low, in comparison with other B-cell lymphomas and normal B-cells (Rossmann et al 2001). Alemtuzumab has been approved for the treatment of CLL that is refractory to FAMP.

\section{Intravenous alemtuzumb}

Alemtuzumab, also called Campath 1-H, was initially investigated in 3 multi-center phase I trials in non-Hodgkin lymphoma (NHL) at doses ranging up to $240 \mathrm{mg}$ per week. Following these early NHL trials, two multicenter phase II trials (protocol 125-K32-005 and 125-K32-009) 
were conducted in Europe and USA, respectively. These trials treated a total of 149 patients with a variety of lymphoproliferative disorders.

Osterborg and colleagues published a report on a subset of 29 patients treated on study 125-K32-005, who had CLL and who had relapsed after an initial response to chemotherapy $(n=8)$ or who were refractory $(n=21)$ (Osterborg et al 1997). Due to the fact that FAMP had been available in Europe only since 1994, only 3 of these 29 patients had been previously treated with FAMP. All patients were treated with alemtuzumab at a dose of $30 \mathrm{mg}$ intravenously over 2 hours 3 times per week (TIW) for a maximum of 12 weeks. Among the 8 relapsed patients, 3 (38\%) showed a response while 9 of $21(43 \%)$ with refractory disease were able to achieve a response using alemtuzumab. It was also shown that CLL cells were rapidly eliminated from blood in $97 \%$ of the patients and a bone marrow CR was obtained in $37 \%$ while splenomegaly resolved in $32 \%$ of patients. On the contrary, lymphoadenopathy resolved in only $7 \%$ of patients. The median response duration was 12 months (range 6-25+). Although World Health Organization (WHO) grade 4 neutropenia and thrombocytopenia developed in $3(10 \%)$ and 2 (7\%) patients respectively, these toxicities resolved in most responding patients during continued alemtuzumab treatment. In all patients a profound lymphopenia $\left(<0.5 \times 10^{3} / \mu \mathrm{L}\right)$ was observed. Bacterial septicemia was detected in 4 patients while 2 patients developed opportunistic infections.

Twenty-four patients with B-cell CLL $(n=23)$ or T-cell prolymphocytic leukemia $(n=1)$ were enrolled in the US phase II trial (125-K32-009) (Rai et al 2002). These patients were treated at 6 US centers with a target dose of $30 \mathrm{mg}$ TIW for up to 16 weeks. Differently from the European trial, all of these patients were previously treated with FAMP and 71\% had either not responded to FAMP or had responded initially but relapsed within 6 months of treatment. The remaining $29 \%$ of patients had been sensitive to a previous treatment with FAMP but when they relapsed they had not responded to subsequent chemotherapy regimens. Partial responses were observed in 8 of 24 patients (33\%). The median time to achieve response was 3.9 months (range 1.6-5.3 months) and the median duration of response was 15.4 months (range 4.6-38.0 months). Median time to disease progression was 19.6 (range 7.7-42 months) and median survival time was 35.8 months (range 8.8 to $\geq 47.1$ months). Antibiotic prophylaxis was not mandated in this study and opportunistic infections were observed in $41.7 \%$ of patients. Pulmonary infections were the most common: there were 3 proven cases of Pneumocystis carinii and 1 suspected; 1 case of fungal infection was also recorded. Opportunistic infections were more common in patients who didn't have clinical beneficial response to monoclonal antibody. Keating and co-workers reported the results of an international study of alemtuzumab in 93 patients with CLL who were refractory to FAMP (Keating et al 2002b). Those patients were treated with intravenous alemtuzumab at a target dose of $30 \mathrm{mg}$ TIW for up to 12 weeks. Also required for entry into this study was prior treatment with an alkylating agent. These were a group of heavily pre-treated patients with the median number of prior regimens being three (range 2-7) while $46 \%$ of patients had received multiple FAMP treatments. Almost half of the patients (48\%) had never responded to any nucleoside analog-based regimen. All patients were required to stay on infection prophylaxis involving TMP/SMZ and famciclovir from day 8 of treatment to at least 2 months after completion of alemtuzumab therapy. The overall response rate (ORR) was 33\% (31/93), with CR achieved in 2\% (2/93). Median time to disease progression was 4.7 months for the intentto-treat population and 9.5 months for responders. Median survival for responders was 32 months, which exceeds the 8- to 9-month median survival time reported for patients who failed FAMP. Lymphocytosis completely resolved in $83 \%$ of patients. Peripheral blood was cleared of malignant lymphocytes in 26 of 28 responders (93\%) and in 34 of 40 patients (85\%) with stable disease. Among the 31 responders, $48 \%$ completely cleared CLL cells from the bone marrow, and an additional $23 \%$ showed a reduction in malignant lymphocytes of at least $50 \%$ in bone marrow. Other major sites of disease also showed marked improvement with alemtuzumab therapy. Splenomegaly and hepatomegaly were completely resolved or reduced by at least $50 \%$ in $82 \%$ and $75 \%$ of patients, respectively. There was an inverse correlation between lymph node size and the likelihood of response. While $91 \%$ of patients whose largest lymph node measured less than 20 mm experienced improvement of at least $50 \%$, only $10 \%$ of patients with lymphnodes greater than $50 \mathrm{~mm}$ achieved more than $50 \%$ reduction in lymph node size. Among patients with cytopenias at baseline, 55\% achieved more than 50\% increase from baseline values, which illustrates improvement in bone marrow function with alemtuzumab treatment. Moreover, the majority of patients experienced resolution of B symptoms and fatigue, including those who did not achieve NCI-WG criteria response (67\%). Infusion-related adverse events were very common but typically of mild or moderate in severity and generally diminished with time. Infections developed in 51 patients (55\%); approximately half were mild to moderate and half were more severe. Grade 3 or 4 infections 
developed in significantly more patients who did not respond to alemtuzumab (22 of 62 non-responders) than in responders (3 infections in 31 responders; $p<0.01$ ). Unlike the early studies with alemtuzumab in CLL cited above, prophylaxis against viral infection and Pneumocistis carinii (PCP) was required in this large pivotal trial and made a considerable difference in the incidence of PCP infections. Only 1 case of PCP was reported and this occurred in a patient who did not receive prophylaxis as outlined in the study. Overall 11 patients developed opportunistic infections during the treatment period and another 7 patients developed opportunistic infections in the post-treatment follow-up period. The most commonly reported event in this context was reactivation of CMV occurring in 7 patients, all appearing during the treatment period. Five of the cases resolved and 2 resulted in discontinuation of alemtuzumab with subsequent withdrawal from the study.

The results of treatment with alemtuzumab were also reported in a series of 78 patients with a variety of advanced or refractory chronic lymphoproliferative disorders. Alemtuzumab was given at $30 \mathrm{mg}$ TIW intravenously for a minimum of 4 weeks and a maximum of 12 weeks, depending on response. Pre-medication with acetaminophen and diphenhydramine was required prior to each infusion and all patients were given prophylactic antibiotic coverage with TMP/SMZ and valacyclovir (Ferrajoli et al 2003).

CLL was the most common $(n=42)$ diagnosis in this series of 78 heavily pre-treated patients who had received a median of three (range 1-9) prior therapies. Among these 42 patients with CLL, 19 were considered sensitive to, while 23 were considered refractory to, FAMP. Among the subgroup of patients with CLL, there was a 31\% (13/42) ORR with CR occurring in 2 patients (5\%), PR in 10 patients $(24 \%)$ and nodular PR in 1 patient (2\%). Of the 19 patients with CLL that were "FAMP sensitive", 2 CR and 5 PRs were achieved for an ORR of $37 \%$. In those 23 patients who were considered "FAMP resistant", there were no CRs and 6 PRs for an ORR of $26 \%$.

As in previous studies clearance of blood lymphocytosis was achieved in a large proportion (84\%) of the total patient population while nearly half the patients (49\%) achieved resolution of the bone-marrow disease. A decrease in the size of enlarged spleen and liver by $50 \%$ or more occurred in $56 \%$ and $59 \%$ of patients, respectively, while lymphadenopathy decreased by at least $50 \%$ in $39 \%$ of patients. Seventy-one per cent of CLL patients developed proven or clinically suspected infection. CMV reactivation was the most common viral infection, occurring in $29 \%$ of 42 CLL patients.
All of these patients responded to intravenous ganciclovir or foscarnet. Other infectious toxicities reported for the entire group of 78 patients were 17 episodes of bacteremia in 11 patients, 11 episodes of pneumonia in 10 patients, 3 cases of herpes virus infections, and 1 case of invasive aspergillosis.

In this study also, infusion-related adverse events were common. Fever occurred in $85 \%$ and rigors in $42 \%$ of patients. Other immediate events included rash (42\%), nausea (35\%), dyspnea (31\%), hypotension (18\%), and headache (7\%). Cardiovascular toxicity was seen in 3 patients (chest pain but no ischemic heart disease, congestive heart failure, depressed systolic ejection fraction at echocardiographic evaluation), all of whom had T-cell malignancies. Hematological toxicity included grade $3(19 \%)$ and grade $4(15 \%)$ neutropenia while grade 3 and 4 thrombocytopenia occurred in $28 \%$ and $13 \%$, respectively. Persistent lymphopenia resulted in all patients.

Several additional phase II studies have been performed in FAMP-refractory patients. In one trial by McCune et al (2002), 23 heavily pretreated patients with relapsed or refractory CLL or prolymphocytic leukemia were treated with alemtuzumab $30 \mathrm{mg} 3$ times weekly for up to 12 weeks. All of the $18 \mathrm{~B}-\mathrm{CLL}$ patients had failed FAMP therapy within 6 months prior to initiation of this study. Nine of 17 evaluable patients (53\%) had an objective response, including 6 CR (35\%).

In a recently published study by Moreton et al (2005), 91 patients with relapsed or refractory CLL (of which $48 \%$ were refractory to purine analog therapy) were treated with alemtuzumab with the goal of eradicating detectable disease using minimal residual disease (MRD) flow cytometry. After a median 9 weeks of treatment, 32 patients (35\%) achieved CR and 17 patients (19\%) reached PR according to NCI-WG criteria. MRD flow cytometry demonstrated blood and bone marrow MRD negativity in 18 responding patients (20\%; among $12 \mathrm{CR}$ and $6 \mathrm{PR}$ ). Median survival for patients who had achieved MRD-negative CR was not reached, compared with 60 months for patients with MRD-positive CR, 70 months for patients with PR, and 15 months for non-responders (NRs) $(p=0.0007)$. Treatment-free survival was significantly longer for patients achieving a MRD-negative CR (not reached), compared with those with MRD-positive CR (20 months), PR (13 months), and non-responders (6 months) ( $\mathrm{p}<0.0001)$. The OS of patients achieving MRD-negative CR was $84 \%$ at 60 months. The absence of lymphadenopathy correlated well with achieving MRD negativity: 13 of 33 patients 
(39\%) who were free from lymphadenopathy achieved MRD-negative CR while none of the patients with bulky lymphadenopathy (less than $50 \mathrm{~mm}$ ) achieved MRD-negative status. This study shows that treatment with alemtuzumab can lead to undetectable levels of MRD, which translates to longer OS and treatment-free survival among patients with relapsed/refractory CLL. Rigors and fever were the most common adverse events occurring in $76 \%$ of patients and were more frequently grade 1 or 2 in severity. Less frequent adverse events included fatigue (11\%), dyspnea $(4 \%)$, headache $(4 \%)$, dizziness $(3 \%)$, bronchospasm $(2 \%)$, and diarrhea (2\%). Adverse events declined in frequency by the end of week 3 of therapy. Neutropenia below $1.0 \times 10^{9} / \mathrm{L}$ occurred in $43(48 \%)$ patients and below $0.5 \times 10^{9} / \mathrm{L}$ in $27(30 \%)$. Granulocyte colony stimulating factor (G-CSF) was administered to $18(20 \%)$ patients with a median neutrophil count of $0.35 \times 10^{9} / \mathrm{L}$ (range, $0.02-0.6)$ and the neutrophil count rose to a median of $1.15 \times 10^{9} / \mathrm{L}$ (range, 0.5-9.2). Thrombocytopenia occurred in $65(73 \%)$ and was less than $50 \times 10^{9} / \mathrm{L}$ in $41(46 \%)$. Thirty-nine patients (43\%) experienced 1 or more infectious episodes during or within 1 month of completing alemtuzumab therapy. There were 19 mild (grade 1 or 2) infectious episodes and 33 severe (grade 3 or 4) episodes. A total of $8(8 \%)$ patients developed $\mathrm{CMV}$ reactivation at a median of 34 days after the start of therapy (range 14-58). One patient died from CMV pneumonitis and after this, screening with pre-emptive therapy for CMV reactivation was instituted. All cases of CMV reactivation detected on screening resolved on anti-viral therapy. There were 31 documented infections in the period following alemtuzumab among 21 (23\%) patients (excluding infections occurring during neutropenia following subsequent stem cell transplantation). Infections following the cessation of alemtuzumab in non-MRD-negative CR patients occurred after a median of 9 months (range 1-41) and in the MRD-negative patients after a median of 3 months (range 1-12).

\section{Subcutaneous alemtuzumab}

Because of the virtual certainty of infusion-related reactions which occur at a very high frequency, even when patients are pre-treated with antihistamines and acetaminophen, investigators have embarked upon studies to determine if alemtuzumab can be made more user-friendly when it is administered via the subcutaneous route.

The German CLL Study Group initiated the CLL2H trial to evaluate the subcutaneous (SC) application of alemtuzumab $30 \mathrm{mg}$ TIW for a maximum of 12 weeks in fludarabine-refractory CLL patients (Stilgenbauer et al 2004). An intravenous dose-escalation schedule of 3, 10, and $30 \mathrm{mg}$ was used and patients were then switched to the subcutaneous route of administration. This trial essentially duplicated the 'pivotal' trial conducted by Keating et al (2002b) but replaced the intravenous route with the subcutaneous route of administration. In an interim analysis of the first 50 patients enrolled, there were 4\% CR, 33\% PR, 44\% stable disease, and 18\% progressive disease. The median OS at the time of the report was 17.4 months and median progression-free survival was 10.8 months. These results compare favorably with the Keating trial. Response rates similar to the overall study population were observed in patients with prognostic genetic subtypes (ie, deletions of $17 \mathrm{p}, 11 \mathrm{q}$, and unmutated $\mathrm{V}_{\mathrm{H}}$ genes).

The UKCLL02 study assessed the safety and efficacy of SC alemtuzumab in FAMP-refractory CLL (Sayala et al 2006). Subcutaneous alemtuzumab was given at a dose of $30 \mathrm{mg}$ TIW for up to 24 weeks depending on 6-weekly marrow assessments. Patients failing to respond to alemtuzumab could receive oral FAMP ( $40 \mathrm{mg} / \mathrm{m}^{2} /$ day for 3 days every 4 weeks) combined with SC alemtuzumab. Of 53 patients (median age 64, range 41 to 79 ) enrolled, 50 patients have completed therapy and 49 were evaluable. Responses to alemtuzumab monotherapy $(\mathrm{n}=$ 49) were 7 CR (5 MRD-, 2 MRD+), 15 PR (1 MRD-), 25 NR, and 2 patients died on treatment. Alemtuzumab was given for a median of 18.8 weeks (range 1.6-24) with a median dose of $1370.5 \mathrm{mg}$ (range 106-2323 mg). Seventeen patients (6 PRs and 11 NRs) received concurrent FAMP and alemtuzumab SC ( 2 courses FAMP median [range 1-3]). Two NR achieved a PR and $1 \mathrm{PR}$ achieved a CR (MRD positive). Therefore the overall response rate for the whole cohort was 24/49 (49\%) including $6 \mathrm{MRD}$ negative patients (5 CRs and $1 \mathrm{PR}$ ). 22/38 patients (58\%) with poor risk deletions (11q-and/or 17p-) and/or p53 dysfunction responded to treatment, including 5 patients who achieved MRD-negative CRs, 3 MRD-positive CR, and 14 PR. Serious infections during alemtuzumab monotherapy were: CMV reactivation (18), febrile neutropenia (10), invasive fungal infection (4), pneumonia (7), and septicemia (2); on the combination, CMV reactivation (3 cases) and septicemia in (1). All CMV reactivations resolved on antiviral therapy. Grade 3+ thrombocytopenia and neutropenia was seen in 26 and 41 patients on alemtuzumab monotherapy as well as in 1 and 5 patients on combined therapy, respectively. Hale et al (2004) studied the blood concentrations of alemtuzumab as well as anti-globulin responses following intravenous or subcutaneous routes of administration in CLL patients. 
Subcutaneous alemtuzumab yielded serum concentrations similar to those achieved with intravenous alemtuzumab, although this was achieved with slightly higher cumulative doses. The dominant factor influencing bio-distribution and pharmacokinetics appears to be the extent of tumor burden. Subcutaneous alemtuzumab is more convenient and better tolerated and only rarely may be associated with formation of anti-alemtuzumab antibodies, particularly in those patients who were previously untreated. The 2 of 31 patients who demonstrated anti-alemtuzumab antibody $(3.7 \times 106 \mathrm{U} / \mathrm{mL}$ in 1 case and $4800 \mathrm{U} / \mathrm{mL}$ in the second one) on subcutaneous treatment, unlike the other patients, did not show significant reductions in lymphocyte counts but had marked local skin reactions which did not diminish with continued therapy. All 30 patients tested for anti-antiglobulins after intravenous treatment with alemtuzumab were below the limit of detection $(488 \mathrm{U} / \mathrm{mL})$.

A reduced dose of $10 \mathrm{mg}$ subcutaneous alemtuzumab has been tested in a small series of patients with refractory CLL (Cortelezzi et al 2005). Sixteen patients were enrolled. They had received a median of 3 prior lines of chemotherapy, all were refractory to alkylating agents, 14 were refractory to FAMP, while 2 were not allowed purine analogs containing regimen due to previous Coombs' positive anemia. Half of the patients were also refractory to rituximab-containing regimens. All received at least 4 weeks of alemtuzumab, 12 patients completed all 18 weeks of treatment.

The OR rate, according to NCI-WG criteria was 50\%, including $25 \% \mathrm{CR}$. An objective response was documented in $50 \%$ of patients refractory to both alkylators agents and FAMP. Treatment was well tolerated.

These studies demonstrate that subcutaneous alemtuzumab, a more convenient alternative to intravenous, is safe and appears to have similar efficacy and an improved side-effect profile compared with intravenous alemtuzumab. In addition, according to the Swedish study (Lundin et al 2002), the subcutaneous route of administration may reduce healthcare costs.

Results reported with alemtuzumab administered as monotherapy are summarized in Table 1.

\section{Chemo-immunotherapy with alemtuzumab}

Combination with other drugs and/or monoclonal antibodies has being actively investigated to improve FAMP efficacy. One of the first indications of the activity of alemtuzumab in combination with FAMP came from a small study of 6 patients with CLL refractory to either alemtuzumab or
FAMP as single agents (Kennedy et al 2002). Five of the 6 patients responded, including $1 \mathrm{CR}$, and complete morphologic marrow responses were seen in another 3 patients with eradication of flow-cytometry-measurable disease in 2 of them. Furthermore, 5 patients were alive at the 12-month follow-up. Toxicity during combined was acceptable with only 1 patient requiring hospital admission with Pseudomonas bronchopneumonia during neutropenia.

Elter et al pursued the FAMP plus alemtuzumab combination further. FAMP was given at $30 \mathrm{mg} / \mathrm{m}^{2}$ intravenously daily on days $1-3$, which was immediately followed by alemtuzumab at $30 \mathrm{mg}$ given over 2 hours for 3 doses on days 1-3 as well (FluCam regimen). Courses were repeated every 4 weeks for a total of 6 rounds. In addition to evaluating clinical responses, MRD was measured using 4-color flow cytometry (Elter et al 2005). A total of 36 patients were treated in this phase II study, median age was 61 years, mean number of previous treatment was 2.6. Twenty-two patients had previously been treated with FAMP and 9 of them (41\%) were refractory to FAMP treatment. Seventy-eight per cent of patients presented a Binet Stage C. All patients had extensive bone marrow infiltration at baseline and the majority had lymphoadenopathy and/or splenomegaly. The ORR was $83 \%$ according to NCI-WG criteria with 11 patients achieving a CR (30\%) and 19 patients achieving PR (53\%). Eight of the 12 patients who were refractory to prior therapy responded (4 CRs and 4 PRs). The majority of 22 patients pretreated with FAMP-containing regimen showed a good response to FluCam (6 CRs, 12 PRs). Of the 9 patients who had been refractory to FAMP, 6 responded to treatment. The lymphocytosis present at baseline resolved rapidly in most patients during the first 2 cycles of therapy. The median OS for all patients was 35.6 months, time to progression (TTP) of 12.97 months. For patients achieving a CR the median OS was not reached and the median TTP was calculated as 21.9 months. Acute infusion-associated adverse effects (fever, chills, and skin reactions) were mild (grade 1 or 2 CTC) in the majority of patients and were observed predominantly in association with initial alemtuzumab dose. The major toxicity in 140 assessable cycles was myelotoxicity with 44\% grade 3-4 leukopenia, 26\% grade 3-4 neutropenia and 305 grade 3-4 thrombocytopenia. There were only 2 CTC grade 3 subclinical CMV reactivations. Two patients showed fungal pneumonia due to Aspergillus fumigatus. One patient died from an Escherichia coli septic shock.

A phase II study to determine the efficacy and safety of a 4-week combination regimen consisting of FAMP, CTX, and Campath-1H (FCC) in relapsed patients with CLL has been reported (Montillo et al 2007). Patients received FCC 
Table I Responses to alemtuzumab as monotherapy in relapsed/refractory CLL

\begin{tabular}{|c|c|c|c|c|}
\hline Reference & Schedule of treatment & $\begin{array}{l}\text { No.pts } \\
\text { evaluable }\end{array}$ & $\%$ OR/\% CR & $\begin{array}{l}\text { Route of } \\
\text { administration }\end{array}$ \\
\hline Osterborg et al 1997 & $30 \mathrm{mg}$ TIW $\times 12 \mathrm{~W}$ & 29 & $42 / 4$ & iv \\
\hline Rai et al 2002 & $30 \mathrm{mg}$ TIW $\times 16 \mathrm{~W}$ & 24 & $33 / 0$ & iv \\
\hline Keating et al 2002 & $30 \mathrm{mg}$ TIW $\times 12 \mathrm{~W}$ & 93 & $33 / 2$ & iv \\
\hline McCune et al 2002 & $30 \mathrm{mg}$ TIW $\times 12 \mathrm{~W}$ & 17 & $53 / 35$ & iv \\
\hline Ferrajoli et al 2003 & $30 \mathrm{mg}$ TIW $\times 12 \mathrm{~W}$ & 42 & $31 / 5$ & iv \\
\hline Lozanski et al 2004 & $30 \mathrm{mg}$ TIW $\times 12 \mathrm{~W}$ & 36 & $31 / 6$ & iv \\
\hline Stilgenbauer et al 2004 & $30 \mathrm{mg}$ TIW $\times 12 \mathrm{~W}$ & 50 & $37 / 4$ & sc \\
\hline Moreton et al 2005 & $30 \mathrm{mg}$ TIW $\times 16 \mathrm{~W}$ & 91 & $35 / 54$ & iv \\
\hline Cortelezzi et al 2005 & $10 \mathrm{mg}$ TIW $\times 18 \mathrm{~W}$ & 16 & $8 / 4$ & sc \\
\hline
\end{tabular}

Abbreviations: CR, complete response; iv, intravenous infusion; No, number; OR, overall response; pts, patients; TIW, 3 times a weeks; sc, subcutaneous injection;W, week.

after a short period of alemtuzumab dose escalation, from $3 \mathrm{mg}$ to $10 \mathrm{mg}$ to $20 \mathrm{mg}$ on consecutive days. The FCC regimen consisted of FAMP $40 \mathrm{mg} / \mathrm{m}^{2} /$ day PO (PO is OK) (Days 1-3), CTX $250 \mathrm{mg} / \mathrm{m}^{2} /$ day PO (Days 1-3), followed by alemtuzumab $20 \mathrm{mg}$ SC (Days 1-3). This combination was repeated on day 29 for up to 6 cycles. MRD was measured by 4-color flow cytometry and consensus primer PCR. Twentythree patients were enrolled. The median age of the patients was 57.0 years (range, 42-79), 13/23 (56\%) were male, 21/23 ( $91 \%$ ) had Binet stage B and C disease, and the median number of prior treatment regimens was 2 (range, 1-4). Among the 18 evaluable patients, ORR was 79\%, with 7 (37\%) patients achieving CR, 7 (37\%) PR, while 1 (5\%) patient a PRn. Three patients had stable disease, while 1 showed progressive disease. MRD negativity was achieved in the bone marrow of 4/14 (27\%) patients. Grade III-IV neutropenia episodes were observed in $43 \%$ of the administered courses while grade IIIIV thrombocytopenia episodes were detected only in $8.0 \%$ of cycles. Four major infections were recorded: 2 sustained by Mycobacterium tuberculosis (1 cutis, 1 lung), 1 by Nocardia (lung), and 1 by E. coli (sepsis). CMV reactivation occurred in 6 patients: no CMV disease was recorded.

\section{Monoclonal antibodies in combination}

A main rationale for using monoclonal antibodies in combination is to overcome some of the limitations of single-agent monoclonal antibodies use (Nabhan and Rosen 2002). These include: a) variable expression of CD20 and CD52 on CLL cells of individual patients; b) synergistic activity in anatomic compartments such as lymphnode sites (where rituximab is expected to be more effective), versus marrow, (better clearance of malignant lymphocytes with alemtuzumab); c) engagement of distinct intracellular signaling pathways resulting in apoptotic cell death.
Faderl et al (2003) conducted an exploratory study of alemtuzumab plus rituximab in patients with relapsed and refractory chronic lymphoid malignancies. The schedule was empirically derived from established single-agent monoclonal antibody experience. Rituximab was given at $375 \mathrm{mg} / \mathrm{m}^{2}$ weekly for 4 weeks with alemtuzumab at $30 \mathrm{mg}$ intravenously twice weekly during weeks $2-4$, preceded by a dose escalation of 3,10 , and $30 \mathrm{mg}$ on 3 consecutive days during week 1. Patients received standard pre-medication and anti-infectious prophylaxis. Forty-eight patients were enrolled: 32 CLL, 9 CLL/prolymphocytic leukemia (PLL), 1 PLL, 4 mantle-cell leukemia (MCL), 2 Richter transformation. Their median age was 62 years (range 44-79). Patients had received a median of 4 (range 1-9) prior therapies, 39 (79\%) had Rai stage $\geq 3$ disease, and $26(5 \%)$ were refractory to FAMP. The OR rate after a 4-week course of therapy was $52 \%$, including a $\mathrm{CR}$ rate of $8 \%$. Among 32 patients with CLL, the OR was 63\%. Treatment-related adverse events included expected infusion-related side effects (fever, skin reactions, fatigue, nausea, and vomiting), and infectious complications. About half of the patients experienced at least 1 infectious episode, including 7 patients (15\%) with symptomatic CMV reactivation who responded to CMVdirected therapy.

A different schedule was investigated in a phase-I study (Nabhan et al 2004). Rituximab was given at the standard dose of $375 \mathrm{mg} / \mathrm{m}^{2}$ on weeks $1,3,4$, and 5 and alemtuzumab at $3 \mathrm{mg}$ (first cohort), $10 \mathrm{mg}$ (second cohort), and eventually $30 \mathrm{mg}$ (third cohort) 3 times weekly during weeks $2-5$. Twelve patients were enrolled; all had failed prior purineanalog-based therapy. One patient achieved PR whereas all remaining patients had stable disease. Treatment was well tolerated, with most toxicities related to the infusion of alemtuzumab. No opportunistic infections, particularly no CMV reactivation, were recorded. 
In an effort to further optimize dose, schedule, and route of administration, obtain data about soluble CD52 serum levels and their association with prognosis, and gain easy access to subcutaneous alemtuzumab, the MD Anderson Cancer Center group investigated a continuous intravenous infusion (CIV) followed by SC injection of alemtuzumab plus rituximab (Faderl et al 2006). rituximab was given at $375 \mathrm{mg} / \mathrm{m}^{2}$ intravenously IV on day 1 followed by 500 $\mathrm{mg} / \mathrm{m}^{2}$ on days 8,15 , and 22 , and alemtuzumab at $15 \mathrm{mg}$ by continuous intravenous infusion daily for 6 days (days 2-7) followed by $30 \mathrm{mg}$ SC twice weekly on day 3 and 5 of weeks 2-4. Pre-medications and anti-infectious prophylaxis remained identical to those in previous regimens. Maximum duration of therapy was 3 courses. Forty-five patients were enrolled of whom 32 patients were evaluable (30 CLL, 1 CLL/SLL, 1 MZL). Six patients were too early and 7 were inevaluable. The median age was 59 years (range 39-78), the median number of prior therapies was 3 (1-8), and the median $\beta_{2} \mathrm{M} 3.8 \mathrm{mg} / \mathrm{dL}$ (1.7-13.6). Seventeen patients (53\%) had Rai stage $\geq 3$ disease. Eighteen patients (56\%) were refractory to FAMP and alkylators. All patients had prior rituximab; only 4 (13\%) had received alemtuzumab. Sixteen patients (50\%) responded: 8 CR (25\%), 1 PRn (3\%), 7 PR (23\%) following 1 course. Response by site: peripheral blood 24/26 (92\%), marrow 22/32 (69\%), lymph nodes $13 / 26(50 \%)$, and liver/ spleen $8 / 9$ patients $(89 \%)$. The combination was well tolerated with no unexpected toxicities. Most non-hematologic adverse events were infusion-related and $\leq$ grade 2 by NCI toxicity criteria. Fevers and chills occurred in up to $56 \%$ of patients, fatigue in $34 \%$, skin rashes in $19 \%$, nausea in $16 \%$, myalgias in $9 \%$, and diarrhea in $6 \%$. Although toxicites were more frequent with CIV alemtuzumab than during the SC injections, CIV alemtuzumab was well tolerated. CMV reactivation occurred in 7 patients $(22 \%)$ and was the most common infectious complication.

Both combination, chemotherapeutic agents and monoclonal antibodies, have been tested by the CFAR regimen (CTX $250 \mathrm{mg} / \mathrm{m}^{2}$ days 3-5, FAMP $25 \mathrm{mg} / \mathrm{m}^{2}$ days $3-5$, alemtuzumab $30 \mathrm{mg}$ days $1 / 3 / 5$, and rituximab 375-500 $\mathrm{mg} / \mathrm{m}^{2}$ day 2) in 74 patients with advanced CLL (Wierda et al 2006). All patients received PCP and herpesviral prophylaxis, and the incidence of major infections (13\% of patients) was not significantly different from the historical experience with FAMP+CTX+rituximab (FCR). CMV reactivation occurred in $20 \%$ of patients, but was successfully treated in all but one; this patient died with multiple other infections. Of the 74 patients who received CFAR, 65\% responded with complete response in $24 \%$. Responses in patients with unfavorable cytogenetics (17p del, 11q del, complex and 6q del) included $14 \% \mathrm{CR}$ and $50 \% \mathrm{PR} ; 44 \%$ of pts with $17 \mathrm{p}$ del responded.

Results obtained with alemtuzumab in combination are summarized in Table 2.

\section{Alemtuzumab and p53 mutation}

Of all prognostic factors examined in CLL, del(17p13.1), the chromosomal location of the tumor suppressor gene $\mathrm{p} 53$, has a superior predictive value for poor response to conventional therapy (Döhner et al 1995).

Stilgenbauer and colleagues were the first to report a complete response in a single patient with $\operatorname{del}(17 \mathrm{p} 13.1)$ receiving alemtuzumab (Stilgenbauer and Dohner 2002).

More recently Lozanski et al (2004) reported the results observed in a cohort of 36 CLL patients (81\% FAMPrefractory) - 15 (42\%) of whom had P53 mutations or deletions - treated with intravenous alemtuzumab. Mutations of the p53 gene were assessed by amplifying each $\mathrm{p} 53$ exon (5-9) individually from the genomic DNA. Clinical responses were noted in 6 of 15 patients (40\%) with P53 mutations and/or deletions, versus 4 of 21 patients (19\%) without P53 abnormalities. The median response duration for this subset of patients was 8 months (range 3-17 months).

As previously reported, the interim analysis of the CLL2H trial confirm the efficacy of alemtuzumab in patients with p53 abnormalities (Stilgenbauer et al 2004). Moreover, one other retrospective study have confirmed these responses in high-risk patients with p53 mutation (Osuji et al 2005). More importantly, one study examining alemtuzumab as initial therapy or in the relapsed setting given together with high-dose meyhylprednisolone confirmed efficacy of alemtuzumab in CLL patients with p53 mutation (Pettitt et al 2006).

These studies suggest that alemtuzumab therapy may be effective in a subgroup of patients with high-risk cytogenetic markers, which points to a potentially unique mechanism of action for alemtuzumab that is likely independent of a p53mediated pathway.

\section{Safety and tolerability}

Recently, management guidelines for use of alemtuzumab in CLL have been published (Keating et al 2004). In approximately $70 \%-80 \%$ of patients, initial infusions of alemtuzumab are associated with rigors, fever, rash, nausea and, less frequently, dyspnea and hypotension. Fever commonly begins 5-6 hours after the start of infusion. All of the above symptoms are of grade 1 and 2 severity in the majority of 
Table 2 Responses to alemtuzumab in combination with chemotherapy or monoclonal antibodies in relapsed/refractory CLL

\begin{tabular}{|c|c|c|c|}
\hline Reference & Schedule of treatment & No. pts evaluable & $\%$ OR/\% CR \\
\hline Kennedy et al 2002 & $\begin{array}{l}\text { FAMP } 25 \mathrm{mg} / \mathrm{m}^{2} \times 3 \mathrm{~d} \text { Campath } 30 \\
\text { iv } \mathrm{mg} \times 3 \mathrm{~d} / \mathrm{W} \text { every } 28 \mathrm{~d}\end{array}$ & 6 & $83 / 17$ \\
\hline Faderl et al 2003 & $\begin{array}{l}\text { Rituximab } 375 \mathrm{mg} / \mathrm{m}^{2} / \mathrm{W} \times 4 \mathrm{~W} \\
\text { Campath } 30 \mathrm{mg} \text { iv } \times 3 \mathrm{~cd} \times \mathrm{IW} 30 \\
\mathrm{mg} \text { iv } \times 2 \mathrm{~d} / \mathrm{W} \times 2 \mathrm{~W} \times 2 \text { courses }\end{array}$ & 32 & $63 / 6$ \\
\hline Nabhan et al 2004 & $\begin{array}{l}\text { Rituximab } 375 \mathrm{mg} / \mathrm{m}^{2} / \mathrm{WW} \text { I-3-4-5 } \\
\text { Campath 3-0-30 mg iv } \times \text { TIWW 2-5 }\end{array}$ & 12 & $8 / 0$ \\
\hline Montillo et al 2007 & $\begin{array}{l}\text { FAMP } 40 \text { os } \mathrm{mg} / \mathrm{m}^{2} / \mathrm{d} \times 3 \mathrm{~cd} \text { CTX } \\
250 \text { os } \mathrm{mg} / \mathrm{m}^{2} / \mathrm{d} \times 3 \mathrm{~cd} \text { Campath } 10 \\
\mathrm{mg} \mathrm{sc} \times 3 \mathrm{~cd} \text { every } 28 \mathrm{~d} \times 6 \text { courses }\end{array}$ & 18 & $79 / 34$ \\
\hline Elter et al 2005 & $\begin{array}{l}\text { Campath } 30 \text { iv sqm }{ }^{2} \times 3 \text { d FAMP } 30 \\
\mathrm{mg} / \mathrm{m}^{2} \times 3 \text { d every } 28 \mathrm{~d} \times 6 \text { courses }\end{array}$ & 36 & $83 / 30$ \\
\hline Faderl et al 2006 & $\begin{array}{l}\text { Campath } 15 \mathrm{mg} \text { civ } 24 \mathrm{~h} \times 6 \mathrm{~cd} 30 \\
\mathrm{mg} \mathrm{sc} \times 2 \mathrm{~d} / \mathrm{W} \times 3 \mathrm{~W} \text { Rituximab } \\
375 \mathrm{mg} / \mathrm{m}^{2} \mathrm{~d} \text { I } 500 \mathrm{mg} / \mathrm{m}^{2} \mathrm{~d} 8 \mathrm{~d} \mathrm{I} 5 \\
\mathrm{~d} 22 \times 3 \text { courses }\end{array}$ & 32 & $50 / 25$ \\
\hline Wierda et al 2006 & $\begin{array}{l}\text { CTX } 250 \mathrm{mg} / \mathrm{m}^{2} \text { days } 3-5 \text {, FAMP } \\
25 \mathrm{mg} / \mathrm{m}^{2} \text { days } 3-5 \text {, Campath } 30 \\
\mathrm{mg} \text { days } \mathrm{I}, 3,5, \text { Rituximab } 375-500 \\
\mathrm{mg} / \mathrm{m}^{2} \text { day } 2 \times 6 \text { courses }\end{array}$ & 74 & $65 / 24$ \\
\hline
\end{tabular}

Abbreviations: $c d$, consecutive days; civ, continuous intravenous infusion; $C R$, complete response; $d$, days; No, number; iv, intravenous infusion; OR, overall response; os, oral; pts, patients; sc, subcutaneous injection;TIW, 3 times a weeks; W, week.

patients, and are most common in the first week of therapy, diminishing rapidly thereafter. Prophylactic use of antihistamines and acetaminophen, slowing of the infusion rate and, severe cases, limited use of steroids can largely abrogate these side effects.

Subcutaneous route of administration is a more convenient alternative to alemtuzumab intravenous, is safe and appears to have similar efficacy improving side-effect profile compared with intravenous alemtuzumab.

Blood concentrations of IV or SC alemtuzumab have been measured in patients included in various clinical trials (Hale et al 2004). The highest measured blood concentrations of alemtuzumab were similar for IV administration $(<0.5-18.3 \mu \mathrm{g} / \mathrm{mL}$, mean $5.4 \mu \mathrm{g} / \mu \mathrm{L})$ and for SC administration (0.6-24.8 $\mu \mathrm{g} / \mathrm{mL}$, mean $5.4 \mu \mathrm{g} / \mathrm{mL})$. The estimated half-life of alemtuzumab was 9 days for both modes of administration.

Hematologic side effects can occur in patients treated with alemtuzumab. Thrombocytopenia occurs most commonly during weeks 2-4 and neutropenia during weeks 4-8. In general, these cytopenias are reversible and may be managed effectively by transfusion and growth factor support; discontinuation of therapy is not recommended. Severe lymphopenia, with reduction in both B and T-cells, is the most significant hematologic effect of alemtuzumab.
The ensuing immunosuppression, that often develops rapidly after the first infusion, predisposed patients to infections with the most common being viral (varicella-zoster virus, herpes simplex virus, and CMV). Other infections include those caused by bacterial pathogens as well as opportunistic organisms. Routine use of antibacterial and antiviral prophylaxis in more recent studies have been associated with a decreased incidence of infections (Ferrajoli et al 2003). Guidelines recently published recommend mandatory use of this strategy (Keating et al 2004).

The incidence of infections with alemtuzumab appears to be significantly lower in previously untreated patients (Lundin et al 2002). In this study the recovery of immune cells was slow and the median cell count of all lymphoid subpopulations remained at less than $25 \%$ of the baseline values for greater than 9 months post treatment. Development of an EBV-positive lymphoprolipherative disorder after therapy with alemtuzumab has been reported and may be related to the profound immunosuppression associated with its use (Ghobrial et al 2003).

Recently reports of possible cardiac toxicity of alemtuzumab in patients with mycosis fungoides have been published, but the lack of such reports in the earlier trials, where higher total doses of alemtuzumab were administered, would necessitate further confirmation of any possible direct association (Lenihan et al 2004). 


\section{Cytomegalovirus reactivation}

The infectious toxicities of alemtuzumab have been well documented and are generally prevented with appropriate prophylactic medications. One topic of continued interest and concern is the issue of CMV reactivation. In the pivotal trial by Keating et al (2002b), in relapsed or refractory CLL patients, CMV reactivation occurred in $7.5 \%$ of patients. The typical presentation was fever and antigenemia. Organ involvement did not occur and there were no deaths. The Stanford group had a similar experience (Nguyen et al 2002). Five out of 34 patients with relapsed and refractory CLL treated with alemtuzumab developed fever and CMV antigenemia. All patients had resolution of fever and antigenemia after therapy with ganciclovir. None of the 5 patients developed symptoms or findings suggestive of a CMV-associated clinical syndrome. The Swedish group (Lundin et al 2002) reported CMV reactivation (verified by polymerase chain reaction) following alemtuzumab therapy among 4 out of $41(10 \%)$ previously untreated CLL patients. All 4 had fever without pneumonitis. These events occurred after 4, 5, 11, and 12 weeks of alemtuzumab therapy, respectively. Three patients received intravenous ganciclovir treatment and responded promptly. One patient recovered spontaneously. In 2 of these 4 cases, alemtuzumab treatment was restarted while the patient received oral ganciclovir prophylaxis without further CMV problems, The German CLL Study Group studied consolidation with alemtuzumab in patients with CLL in first remission in a phase III randomized trial (Wendtner et al 2004). After a median of 4 weeks, alemtuzumab treatment was interrupted due to serious infections in 7 of 11 patients. Four patients showed CMV reactivation detected by CMV-specific PCR and required intravenous ganciclovir treatment because of rising viral load combined with fevers or because of CMV pneumonia. CMV pneumonia was diagnosed in 2 patients who had increased CMV titer by CMV-specific PCR, positive CMV antigen, radiological signs of pneumonia and clinical findings of fever, dyspnea and coughing. Ganciclovir resolved the CMV symptoms and CMV-DNA declined to baseline levels by week 8 in all treated patients. The clinical significance of detection of CMV by plasma DNA PCR in patients with CLL treated with alemtuzumab remains uncertain, but this test provides an objective measure of CMV activity and may be diagnostic in patients with unexplained fever.

Updated guidelines providing recommendations for the management of CMV reactivation have been recently published (O'Brien et al 2006). This new suggested approach to this topic is more aggressive incorporating surveillance/preemptive therapy and up-front prophylaxis .
Nearly half of patients with treatment-naïve CLL receiving alemtuzumab therapy can present with asymptomatic CMV infection. Incidence of symptomatic infection among patients with previously untreated CLL is approximately $10 \%$ even when a weekly CMV surveillance is ruled out. In contrast, the incidence of symptomatic reactivation among patients with previously treated CLL appears to decrease from approximately $30 \%$ to $9 \%$ with the regular CMV surveillance and preemptive therapy. CMV surveillance might be considered labor-intensive and/or inaccessible for many treatment centers thus the authors suggested up-front prophylaxis might be less labor - intensive because patients are treated with a CMV specific antiviral regimen regardless of their CMV antigenemia status (O'Brien et al 2006).

Based on our experience, in order to reduce an additional risk of myelosuppression and costs issue, up-front prophilaxis should be limited to patients with previous CMV reactivation or disease and/or in case of inaccessibility of surveillance tests, considering this last, together with pre-emptive therapy, as the preferable approach in the majority of patients.

\section{Summary}

The introduction of immunotherapeutic agents such as alemtuzumab is beginning to change the face of CLL treatment. A considerable body of data is emerging through well designed clinical trials that can help guide clinicians to improve outcomes for patients with CLL, including those with advanced or refractory disease. Therapy with alemtuzumab offers a more effective option compared to combination chemotherapy for treatment of patients with CLL who have relapsed or who are refractory to nucleoside analogs. The response rate in patients with advanced disease inversely correlates with the presence and size of the lymph nodes. However, patients not fulfilling the criteria for PR with regard to lymphoadenopathy may have tumor reduction in the bone marrow, with improvements in disease-related symptoms and cytopenia. A number of therapeutic strategies are being evaluated to improve this outcome even further. Combination therapy with other immunologic or chemotherapeutic agents may accomplish this goal. alemtuzumab is particularly effective in patients with p53 mutations. Significant efforts are being made to identify patients who will not respond optimally to conventional therapy as patients with $\mathrm{p} 53$ mutations. The earlier use of alemtuzumab in this high-risk patients may produce improved responses while avoiding unnecessary toxicities accompanying suboptimal therapies. Although there are significant potential toxicities associated with 
alemtuzumab, such the infusional reactions and the risk of CMV reactivation, most are manageable. Infusional reactions can be reduced by prophylactic administration of antihistamines and paracetamol or by adding corticosteroids IV during the first week of treatment. Alternatively, SC administration is associated with fewer flu-like symptoms than IV administration. All patients receiving alemtuzumab therapy must be rigorously checked for emerging infections. In particular CMV quantitative polymerase chain reaction or antigenemia should be performed routinely or at least without any delay in all febrile patients, as early administration of valgancyclovir may be very effective.

In conclusion, alemtuzumab is an important drug in the treatment of CLL having an unique properties not shared by chemotherapeutic agents or other monoclonal antibodies. It can be incorporated into various treatment modalities (eg, consolidation, combination with chemotherapy, maintenance therapy) to improve clinical outcomes in a wide range of patients with CLL. Additional randomized phase III trials are urgently warranted.

\section{References}

Byrd JC, Stilgenbauer S, Flinn IW. 2004. Chronic lymphocytic leukemia. In: Hematology American Society of Hematology Education Program Book. Washington, DC: American Society of Hematology. p.163-83.

Catovsky D, Richards S, Matutes E, et al. 2007. Assessment of fludarabine plus cyclophosphamide for patients with chronic lymphocytic leukaemia (the LRF CLL4 trial): a randomised controlled trial. Lancet, 370:230-9.

Cheson BD, Bennet JM, Grever M, et al. 1996. National Cancer Institute - sponsored Working Group Guidelines for chronic lymphocytic leukemia: revised guidelines for diagnosis and treatment. Blood, 87:4990-7.

Cortelezzi A, Pasquini MC, Sarina B, et al. 2005. A pilot study of low-dose subcutaneous alemtuzumab therapy for patients with chemotherapy-refractory chronic lymphocytic leukemia. Haematologica, 90:410-12.

Delannoy A, Hanique G, Ferrant A. 1993. 2-Chlorodeoxyadenosine for patients with B-cell chronic lymphocytic leukemia resistant to fludarabine. $N$ Engl J Med, 328:812.

Dohner H, Fischer K, Bentz M, et al. 1995. p53 gene deletion predicts for poor survival and non-response to therapy with purine analogs in chronic B-cell leukemias. Blood, 85:1580-9.

Eichhorst BF, Busch R, Hopfinger G, et al. 2006. Fludarabine plus cyclophosphamide versus fludarabine alone in first line therapy of younger patients with chronic lymphocytic leukemia. Blood, 107:885-91.

Elter T, Borchmann P, Schulz H, et al. 2005. Fludarabine in combination with alemtuzumab is effective and feasible in patients with relapsed or refractory B-cell chronic lymphocytic leukemia: results of a phase II trial. J Clin Oncol, 23:7024-31

Faderl S, Thomas DA, O'Brien S. et al. 2003. Experience with alemtuzumab plus rituximab in patients with relapsed and refractory lymphoid malignancies. Blood, 101:3413-15.

Faderl S, Ferrajoli A, Wierda WG, et al. 2006. The combination of Alemtuzumab [Continuous Intravenous Infusion (civ) followed by subcutaneous injection (sc)] plus Rituximab has activity in patients (pts) with relapsed chronic lymphocytic leukemia (CLL) [abstract] Blood, 108:2827.
Ferrajoli A, O’Brien SM, Cortes JE. et al. 2003. Phase II study of alemtuzumab in chronic lymphoproliferative disorders. Cancer, 98:773-8.

Flinn IW, Neuberg DS, Grever MR, et al. 2007. Phase III Trial of Fludarabine Plus Cyclophosphamide Compared With Fludarabine for Patients With Previously Untreated Chronic Lymphocytic Leukemia: US Intergroup Trial E2997. J Clin Oncol, 25:793-8.

Ghobrial IM, Otteman LA, White WL. 2003. An EBV-positive lymphoproliferative disorder after therapy with alemtuzumab. $N$ Engl J Med, 349:2570-2.

Gilleece MH and Dexter TM. 1993. Effect of Campath-1H antibody on human hematopoietic progenitors in vitro. Blood, 82:807-812.

Greenwood J, Clark M, Waldmann H. 1993. Structural motifs involved in human IgG antibody effector functions. Eur J Immunol, 23:1098-104.

Grever MR, Leiby JM, Kraut EH, et al. 1985. Low-dose deoxycoformycin in lymphoid malignancy. J Clin Oncol, 3:1196-201.

Hale G, Rebello P, Brettman LR, et al. 2004. Blood concentrations of alemtuzumab and antiglobulin responses in patients with chronic lymphocytic leukemia following intravenous or subcutaneous routes of administration. Blood, 104:948-55.

Heit W, Bunjes D, Wiesneth M, et al. 1986. Ex vivo T-cell depletion with the monoclonal antibody Campath-1 plus human complement effectively prevents acute graft-versus-host disease in allogenic bone marrow transplantation. Br J Haematol, 64:479-86.

Johnson S, Smith AG, Loffler H, et al. 1996. Multicentre prospective randomized trial of fludarabine versus cyclophosphamide, doxorubicin, and prednisone (CAP) for treatment of advanced-stage chronic lymphocytic leukaemia. The French Cooperative Group on CLL. Lancet, $347: 1432-8$

Juliusson G, Elmhorn-Rosenborg A, Liliemark J. 1992. Response to 2chlorodeoxyadenosine in patients with B-cell chronic lymphocytic leukemia resistant to fludarabine. $N$ Engl J Med, 327:1056-61.

Keating MJ, Kantarjian H, Talpaz M, et al. 1989. Fludarabine: a new agent with major activity against chronic lymphocytic leukemia. Blood, $74: 19-25$

Keating MJ, O'Brien S, Kantarjian H, et al. 1993. Long-term follow-up of patients with chronic lymphocytic leukemia treated with fludarabine as a single agent. Blood, 81:2878-84.

Keating MJ, O’Brien S, Kontoyannis D, et al. 2002a. Results of first salvage therapy for patients refractory to a fludarabine regimen in chronic lymphocytic leukemia. Leuk Lymphoma, 43:1755-62.

Keating MJ, Flinn I, Jain V, et al. 2002b. Therapeutic role of alemtuzumab (Campath-1H) in patients who have failed fludarabine: results of a large international study. Blood, 99:3554-61.

Keating MJ, Coutre S, Rai K, et al. 2004. Management guidelines for use of Alemtuzumab in B-cell chronic lymphocytic leukemia. Clin Lymphoma, 4:220-7.

Kennedy B, Rawstron A, Carter C, et al. 2002. Campath-1H and fludarabine in combination are highly active in refractory chronic lymphocytic leukemia. Blood, 99:2245-7.

Kipps TJ . 2001. Chronic lymphocytic leukemia and related diseases. In: Williams Hematology. Beutler E, Lichtman MA, Coller BS, Kipps TJ, Seligsohn U (eds). Mc.Graw-Hill. New York, USA, 1163-1194.

Knospe WH, Loeb V Jr, Huguley CM Jr. 1974. Proceedings: Bi-weekly chlorambucil treatment of chronic lymphocytic leukemia. Cancer, 33:555-62.

Lenihan DJ, Alencar AJ, Yang D, et al. 2004. Cardiac toxicity of alemtuzumab in patients with mycosis fungoides/Sezary syndrome. Blood, 104:655-8.

Leporrier M, Chevret S, Cazin B, et al. 2001. Randomized comparison of fludarabine, CAP, and CHOP in 938 previously untreated stage $\mathrm{B}$ and C chronic lymphocytic leukemia patients. Blood, 98:2319-25.

Lozanski G, Heerema NA, Flinn IW, et al. 2004. Alemtuzumab is an effective therapy for chronic lymphocytic leukemia with p 53 mutations and deletions. Blood, 103: 3278-81.

Lundin J, Kimby E, Bjorkholm M, et al. 2002. Phase II trial of subcutaneous alemtuzumab (Campath-1H) as first-line treatment for patients with Bcell chronic lymphocytic leukemia (B-CLL). Blood, 100:768-773. 
McCune SL, Gockerman JP, Moore JO, et al. 2002. Alemtuzumab in relapsed or refractory chronic lymphocytic leukemia and prolymphocytic leukemia. Leuk Lymphoma, 43:1007-1011.

Montserrat E. 2002. Current and developing chemotherapy for CLL. Med Oncol, 19:S11-S19.

Montillo M, Tedeschi A, Miqueleiz S, et al. 2007. Combined fludarabine, cyclophosphamide, and alemtuzumab (FCC), an active regimen for treated patients with chronic lymphocytic leukemia (CLL) [abstract]. Blood, 110:3133.

Moreton P, Kennedy B, Lucas G, et al. 2005. Eradication of minimal residual disease in B-cell chronic lymphocytic leukemia after alemtuzumab therapy is associated with prolonged survival. J Clin Oncol, 23:2971-9.

Nabhan C, Rosen ST. 2002. Conceptual aspects of combining rituximab and Campath-1H in the treatment of chronic lymphocytic leukemia. Semin Oncol, 29 (Suppl 2):75-80.

Nabhan C, Patton D, Gordon LI, et al. 2004. A pilot trial of rituximab and alemtuzumab combination therapy in patients with relapsed and/or refractory chronic lymphocytic leukemia (CLL). Leuk Lymphoma, 45:2269-73.

Nguyen DD, Cao TM, Dugan K, et al. 2002. Cytomegalovirus viremia during CAMPATH-1H therapy for relapsed and refractory chronic lymphocytic leukemia and prolymphocytic leukemia. Clin Lymphoma, 3:105-110.

O'Brien S, Kantarijan H, Estey E, et al. 1994. Lack of effect of 2-chlorodeoxyadenosine therapy in patients with chronic lymphocytic leukaemia refractory to fludarabine therapy. $N$ Engl J Med, 330:319-22.

O'Brien S, Keating MJ, Mocarski ES. 2006. Updated guidelines on the management of Cytomegalovirus reactivation in patients with chronic lymphocytic leukemia treated with Alemtuzumab. Clin Lymphoma Myeloma, 7:125-30

Oken MM, Kaplan ME. 1979. Combination chemotherapy with cyclophosphamide, vincristine, and prednisone in the treatment of refractory chronic lymphocytic leukemia. Cancer Treat Rep, 63:441-7.

Osterborg A, Dyer MJ, Bunjes D, et al. 1997. Phase II multicenter study of human CD52 antibody in previously treated chronic lymphocytic leukemia. European Study Group of CAMPATH-1H Treatment in Chronic Lymphocytic Leukemia. J Clin Oncol, 15:1567-74.

Osuji NC, Del Giudice I, Matutes E, et al. 2005. The efficacy of alemtuzumab for refractory chronic lymphocytic leukemia in relation to cytogenetic abnormalities of p53. Haematologica, 90:1435-6.
Pettitt AR, Matutes E, Oscier D. 2006. Alemtuzumab in combination with high-dose methylprednisolone is a logical, feasible and highly active therapeutic regimen in chronic lymphocytic leukaemia patients with p53 defects. Leukemia, 20:1441-5.

Rai KR, Peterson BL, Appelbaum FR, et al. 2000. Fludarabine compared with chlorambucil as primary therapy for chronic lymphocytic leukemia. N Engl J Med, 343:1750-7.

Rai KR, Freter CE, Mercier RJ, et al. 2002. Alemtuzumab in previously treated chronic lymphocytic leukemia patients who also had received fludarabine. J Clin Oncol, 20:3891-7.

Rossmann ED, Lundin J, Lenkei R, et al. 2001. Variability in B-cell antigen expression: implications for the treatment of B-cell lymphomas and leukemias with monoclonal antibodies. Hematol J, 2:300-6.

Rowan W, Tite J, Topley P, et al. 1998. Cross-linking of the Campath-1 antigen (CD52) mediates growth inhibition in human B- and T-lymphoma cell lines, and subsequent emergence of CD52-deficient cells. Immunology, 95:427-36.

Sayala HA, Moreton P, Jones RA, et al. 2006. Final report of the UKCLL02 Trial: A phase II study of subcutaneous alemtuzumab plus fludarabine in patients with fludarabine refractory CLL (on behalf of the NCRI CLL Trials Sub-Group) [abstract]. Blood, 108:34.

Saven A, Piro LD. 1993. 2-Chlorodeoxyadenosine: a new nucleoside agent effective in the treatment of lymphoid malignancies. Leuk Lymphoma, 10(Suppl):43-9.

Stilgenbauer S, Dohner H. 2002. Campath-1H-induced complete remission of chronic lymphocytic leukemia despite p53 gene mutation and resistance to chemotherapy. $N$ Engl J Med, 347: 452-3.

Stilgenbauer S, Winkler D, Krober A, et al. 2004. Subcutaneous Campath-1H (alemtuzumab) in fludarabine refractory refractory CLL; interim analysis of the CLL2H Study of the German CLL Study Group (GCLLSG) [abstract]. Blood, 104:478.

Wendtner CM, Ritgen M, Schweighofer CD, et al. 2004. Consolidation with alemtuzumab in patients with chronic lymphocytic leukemia in first remission- experience on safety and efficacy within a randomized multicenter phase III trial of the German CLL Study Group (GCLLSG). Leukemia, 18:1093-101.

Wierda W, O’Brien S, Faderl S, et al. 2006. Combined cyclophosphamide, fludarabine, alemtuzumab, and rituximab (CFAR), an active regimen for heavily treated patients with CLL [abstract]. Blood, 108:31. 